\title{
Identifying Stress Level Among Gamers Using Electroencephalogram Signals
}

\author{
Norzaliza MNor', Sheik Dawood Mohamed Rafi'2, Muhammad Arif Othman³ \\ 1,2,3 Department of Computer Science, Kulliyyah of Information \& Communication Technology (KICT), International Islamic \\ University Malaysia, Jalan Gombak, 51300 Kuala Lumpur \\ norzaliza@iium.edu.my, sheikdawood1103@gmail.com,muhammadarifothman97@gmail.com
}

\begin{abstract}
This research is conducted to identify stress level among gamer using Electroencephalogram Machine (EEG). Electroencephalogram machine or better known as EEG machine is a machine used by neuroscientists to read brain signals activity through various number of channels. The brain signals collected from subjects using 19 channels EEG machine which is DABO Machine. The problem in this research study is to find out if game can induce stress. The expected outcome of this research is that brain signal collected from subjects could give enough evidence about the relationship between playing game and stress level in their daily activities. Objective of the research is to design experimental procedure suitable for understanding the bio-signal of subjects inducing stress and to understand the relationship between four basic emotions (Happy, Calm, Fear, Sad) and the emotion while playing the games. In our research methodology, we focus on five difference stages to complete the research. The stages start with the data collection, pre-processing, features extraction classification and lastly analysis. Later, we able to come out with the result of our research about the stress level for the subject. The experiment was conducted by following a standard protocol experiment for EEG machine. This data will be analysed using Mel Frequency Cepstral Coefficients (MFCC) as feature extraction, and multilayer perceptron (MLP) as classifier. The result show that the subject has positive emotion which is calm and happy at the beginning and ending of playing the game. At the beginning, subject only start with demo, so the subject did not feel pressured and at the end we assumed that the subject feel relieved because of ending the game. After certain time playing the game, the subject starting to have negative emotion until the end of the game. This happen because of subject started to feel stress after plays the higher level of the game. Based on the result, we can conclude that game can induce stress among gamers.
\end{abstract}

Keywords-EEG, stress, gamers, MFCC, MLP.

\section{INTRODUCTION}

Stress is a feeling of emotional or physical tension. It can come from any event or thought that makes you feel frustrated, angry, or nervous. Stress is our body's reaction to a challenge or demand. In short bursts, stress can be positive, such as when it helps you avoid danger or meet a deadline [1-2]. But when stress lasts for a long time, it may harm our health.

The problem in this research study is why stress is important to be measured and what is the relationship between four basic emotions (Happy, Calm, Fear, Sad) and the emotion while playing the games. The experiment was conducted to identify stress level among gamers using electroencephalogram (EEG) machine. The brain signals collected from subjects using 19 channels EEG (electroencephalogram) machine. The experiment was conducted by following a standard protocol experiment for EEG machine usage and DASS-21 (depression anxiety and stress scales) questionnaires [1] with extension of SAM (self-assessment manikin) towards subjects.
To complete the analysing process, we used MFCC (Mel Frequency (epstral Coefficients) as a feature extraction, then MLP (multilayer perceptron) as classifier, to get the expected result. We used these methods to analyse the result and compare the accuracy level between these methods. In the last part of our research, we expect the results to show an accuracy of data emotions. The trajectories of the valence $(\mathrm{V})$ and arousal $(\mathrm{A})$ are when $\mathrm{V}=+1$ and $A=+1$ the subject's emotion is happy, when $V=+1$ and $A=-1$ the subject's emotion is calm, when $V=-1$ and $A=+1$ the subject's emotion is fear whereas when $V=-1$ and $A=-1$ the subject's emotion is sad. As the subject's emotion is happy or calm, he is being motivated whereby if the subject's emotion is fear or sad, he is not motivated while performing the tasks given. The outcome expected from this experiment is that brain signals collected could give enough evidence that the kinds of games given could contribute to intrinsic motivation among our subjects.

This research is conducted to identify stress level among gamer using Electroencephalogram Machine (EEG). Electroencephalogram machine or better known as EEC 
machine is a machine used by neuroscientists to read brain signals activity through various numbers of channels [3]. The brain signals collected from subjects using 19 channels EEG (electroencephalogram) machine which is DABO Machine. The experiment was conducted by following a standard protocol experiment for EEG machine. This data will be analysed using MFCC (Mel Frequency Cepstral Coefficients) as feature extraction, and MLP (multilayer perceptron) as classifier. The problem in this research study is to find out whether game can induce stress or not. The expected outcome of this research is that brain signal collected from subjects could give enough evidence about the relationship between playing game and stress level in their daily activities. Hence the paper set out the following objectives: to design experimental procedure suitable for understanding the bio-signal of subjects inducing stress; and to understand the relationship between four basic emotions (Happy, Calm, Fear, Sad) and the emotion while playing the games.

This is crucial because the research will help Computer Scientist to analyse the stress data for gamers based on physiological signals (EEG) using neural network, since the psychological data itself can be biased. But with help from our psychological signal data, we can help them to analyse the data and get a better result. Other than that, to identify either game can induce stress or otherwise, hence, to find the relationship between game and stress level. Besides, this project is to help researches to analyse the stress data for playing game based on physiological signals (EEG) using neural network, since the psychological data (dass21) itself can be biased.

\section{RELATED WORK}

A. EEG-Based Evaluation of Cognitive and Emotional Arousal An experiment to understand how emotions and/or cognitive load vary while coding in $C$ and Python, to gain better understanding on the EEG method, principles and complexities involved with the collection of data and to understand the steps to perform such an experimental study and to assimilate the emotional and cognitive load that affects the brain when performing programming. In the research, a novel method was adopted to measure and quantitatively analyze EEG data in order to evaluate the emotions and cognitive levels of programmers while coding, using the Emotiv headset device and self-reports in an experimental setup. This Pilot research indicated that the Emotiv Epoc device is feasible to conduct a controlled experiment. Following the results of the experiment above, three distinct differences were observed in the cognitive and emotional measures of the programmers [4]. Python programming languages was more preferred than $C$ programming based on the syntax, difficulty level and time taken was also less in Python. The emotions in Python were more positive as compared to $C$ programming. These variations eventually prove that if the EEG data analysis conducted in controlled environment can reveal exceptional conclusion about psycho-physiological activities. However, in this experiment they do not aim to generalize the results and the evaluation are performed based on the pilot nature of the experiment. This particular thesis helped to formulate the steps that need to be followed in order to analyze the EEG data using the noncommercial tools like EEGLAB and MATLAB.

B. MLP-Performance Evaluation for Pattern Recognition in Myoelectric Signal

This experiment is about the evaluation of MLP's performance on myoelectric signal usage for artificial limbs movements. The signals were gathered from BioPatRec signal repository. MLP was used to train original signals and only collect the re-sampled signal by using NetLab. This experiment also conducted an evaluation of results of different situation to gain the best configuration for artificial limbs to move [5]. This paper stated that comparison process also being made between MLP and other algorithms however, MLP works the best with accuracy of $98.3 \%$ by train the original data. The new resampled data also scores $96.7 \%$ of accuracy with 200 of iteration was used. It can be concluded that MLP may be the best algorithms to process myoelectric signals.

C. Using the Depression Anxiety Stress Scale 21 (DASS 21) across cultures

Experiment in this paper focus on validation of DASS-21 usage in across South East Asian countries. There are 2,630 employees were recruited by 4 different researchers from several companies from six countries to cross validate the result in each country. Paper-based questionnaire and data entry have been conducted. The scores of questionnaires were calculated, and cross validation process was being made to obtain the significant pattern of questions residuals. This paper also mentioned about the psychometrics aspect of DASS-21 with respect to Asian demographics background [6]. The result of this experiment also proposed that some of questions in DASS 21 are causing residuals and cross loading. The researchers also stated in this paper that DASS-18 may work better in term linguistic choices of words because of differences in term of perception.

\section{Emotion and Eelectroencephalogram (EEG)}

Electroencephalogram (EEG) device is a diagnostic tool used by the neurologist to record the electrical activity of the brain using numerous electrodes placed on the scalp [3]. The electrical activity produced by the brain cells (neurons activation) and neural circuits can then be captured. These EEG signals can then be used to analyze the student's dynamic emotions and the respective 
precursor emotion. EEG device has been adopted by many researchers in capturing neural activity in milliseconds from the cortical surface [7].

\section{RESEARCH METHODOLOGY}

In our research methodology, we focus on five different stages to complete the research. The stages start with the data collection, pre-processing, features extraction classification and analysis. In the end, we would be able to come out with the result of our research about the stress level for the subject. Figure 1 shows the overall methodology of our research by using the block diagram.

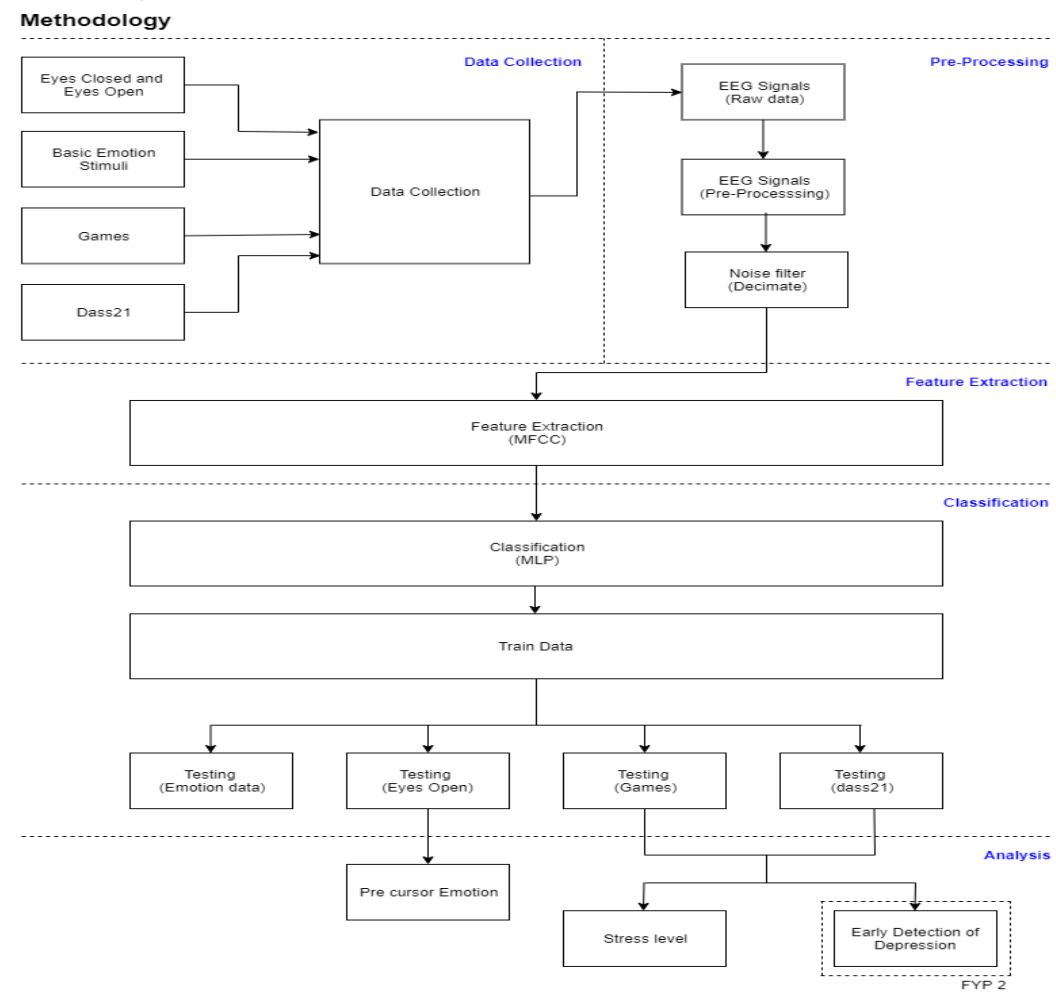

Fig 1. Proposed research methodology block diagram for intrinsic motivation towards games

\section{A. Data Collection}

Data collection is the most important part in our research to ensure that the conclusion that we made from the analysis is correct and useful, it will be the first phase that needs to be completed to obtain the EEG raw signals. There are four stages used in data collection which are eyes closed and eyes open data collection, basic emotion stimuli (IAPS) data collection, games data collection and also DASS-21 data collection. The eyes closed and eyes open data collection is important for the first step in our data collection as it will give the actual data for the subject during the resting state or initial state data before the other task is given, it also will be used to analyze the precursor emotion. The eyes open will be used to represent the emotion calm. Based on our findings, the results from eyes open and close shows the subject's state for that moment and also state a week before.

Next step in the data collection is to collect the EEG raw data in analyzing basic emotion responses of the subject based on four basic emotion stimuli of happy, fear, calm and sad to be used in analyzing the discrete class emotion and also deriving the VA axes for the ASM. Although the ASM requires four quadrants, happy, fear, sad and calm, emotion calm was deriving from the eyes open while emotion happy, fear and sad using IAP's pictures stimuli. Thus, the four chosen basic emotion will be functioned as the basis in our research. Next step will be game data collection. The subject will play 2 games while the brain signal being recorded. This is one of the important parts in the data collection.

Finally, we can check the state of the subject during this part and this plays an important role in the result and progress. For the last data collection task, we will ask the DASS 21 question that consist of 21 question on stress, anxiety and depression to the subject and record their brain signal for all 21 question. Each will be displayed on the screen to the subject, and they are required to answer verbally on the scale of zero to three. The answer of each question is recorded on a paper and to calculate the score of the DASS 21 for each of the subject. 


\section{B. Pre processing}

The EEG raw data need to be pre-processed to ensure all noise and artefact will be reduced. One of the techniques used to reduce noise is elliptic filter.

\section{i. Noise Filter Method (Elliptic Filter)}

The human brain generates electrical signals called EEG signals which are related to body functions. Sources of noise are static electricity or electromagnetic field produced by surrounding devices. In addition to this external noise, the EEG signal can be affected by noise artefact due to body movement example such as facial movement, hand movement and another surrounding artefact.

\section{Feature Extraction (MFCC)}

Initially, features extraction is done to determine the important features of brain signals from all 19 channels of electroencephalogram (EEG) machine. Before second part of the process takes place, which involves classification, we used Mel-Frequency Cepstral Coefficients to extract the features.

\section{Classification (MLP)}

For the classification, we used Multilayer Perceptron (MLP) classifier to classify the brain signals in order to check the intrinsic motivation of the students either being motivated or not. The result is gained by using MLP function readily can be found in MatLab under the type of feedforward artificial neural network.

\section{E. Analysis}

For the analysis part, we will use all the collected data from previous step by using MATLAB to get the result of the stress level of the subject during the experiment. The overall scores for each subject can be considered as preliminary result. This result as well can be used as a comparison to result gained from classification of the patterns.

\section{EXPERIMENTAL DESIGN}

The experimental design used in this experiment is where we place the electrode of the EEG Machine on the head of the subjects based on the 19-channel guide. The placement of the electrode is done manually by attaching the electrode directly to the subject's head without using the special cap. Then we proceed to the next steps of the experiment which is recording the brain signal of the subject during their eyes opened and eyes closed within 1 minute each. Eyes closed data were used to identify the precursor emotion as it represents the initial emotional state [8]. Next, we will do the reaction stimuli where it will be proceeded by viewing the IAPS Emotion Sequence video, consisting of happy, calm, sad, and fear videos for 30 seconds each and record the brain signal along the video were showed. Then, we will ask the subject to play 2 games where each game 5 minutes each. The first game is Chameleon Run game. This game is made up of abstract. The second game is Vector game and this game is somehow related to the reality.

The brain signal will be recorded during the subject play both games. Next, we asked the DASS 21 question that consist of 21 questions on stress, anxiety and depression to the subject and record their brain signal for all 21 questions. Each question will be displayed on the screen to the subject, and they are required to answer verbally on the scale of zero to three. The answer of each question is recorded on a paper to calculate the score of the DASS 21 for each of the subject. Last part of our experimental design will be recording the brain signal of the subject during eyes closed and eyes open within 1 minute each.

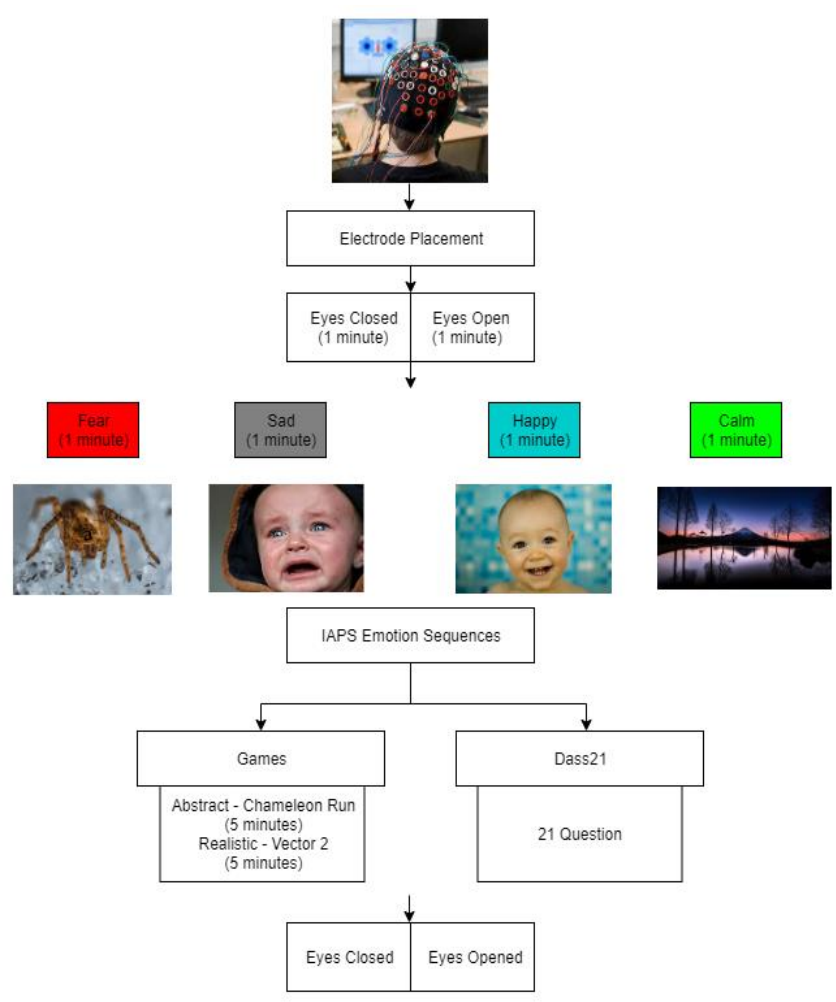

Fig 2. Proposed experimental design

\section{ANALYSIS AND PRESENTATION OF RESULT}

During the progress, we use the data that had been collected about the stress level among gamers using EEG signal as the preliminary result and the methodology that was used to analyse the data is like our proposed methodology in this research. Therefore, by analysing the collected data, we can test the methodology and learn how to analyse the data correctly for our future data. The 
collected data involves one subject aged 23 years old. Below is the result after we analyse the data:

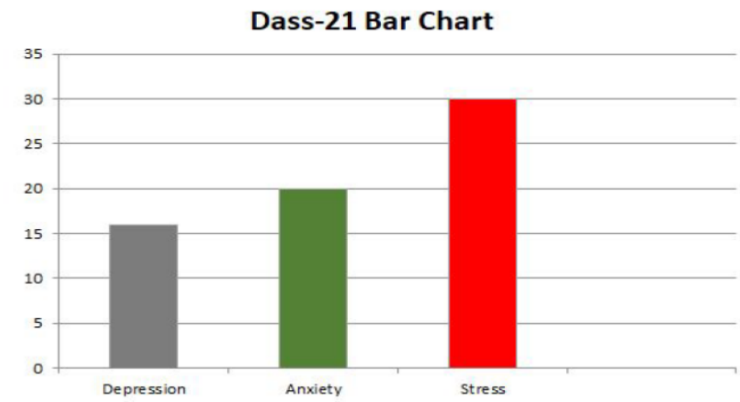

Fig 3. Results of the Subject on Dass21 (Psychological data)

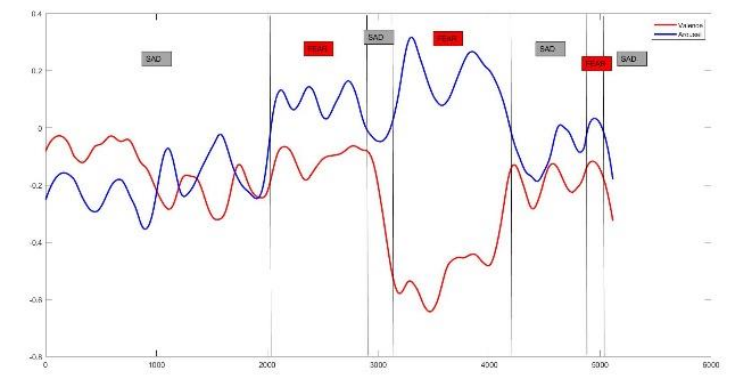

Fig 4. Results of the Subject on Dass21 (Physiological signals)

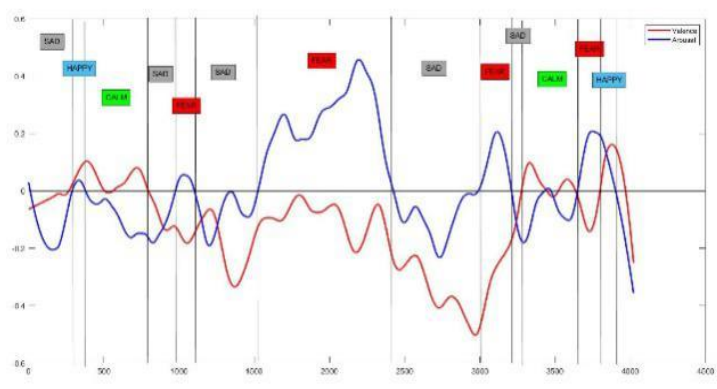

Fig 5. Results of the Subject on Game

Based on the experiment that has been conducted, we manage to calculate the score of the psychological data (DASS 21) for the subject. The DASS 21 questions contain seven questions on depression, stress and anxiety respectively and the score of each question is multiplied by two to get the total score of each category of question. After the total score of each question category is obtained, then we can classify the level of stress, anxiety and depression into five classes which are normal, mild, moderate, severe, and extremely severe.

As we can see in figure 4, the score of depression, anxiety, and stress of the subject is 16,20 , and 30 respectively where we can classify the scores as moderate, extremely severe, and severe. We also record the physiological signals (EEG) of the subject when the subject is answering the DASS 21 questions. The result in figure 4 show that subject only have negative emotion which is fear and sad during the session. Next when we compare the psychological data and the physiological signals, we find that the result is tally in terms of stress level of the subject. We can conclude that the subject was totally honest when answering the DASS21 question. As we can see in figure 5 , the result shows that the subject has only positive emotion which is calm and happy at the beginning and ending of playing the game. Initially when the subject only starts with demo, so that they did not feel pressured whereas at the end we assumed that the subject feels relieved because the game ends. After a period playing the game, the subject starts to have negative emotion until the end of the game. This happens because the subject starts to feel stress after playing the higher level of the game. Based on the result, it is concluded that game can induce stress among gamers.

\section{Future Work}

On future works, we are going to analyse the data using others classifier method instead of multilayer perceptron (MLP) and using others feature extraction method instead of Mel Frequency Cepstral Coefficients (MFCC), then compare the results of the method used to get better evaluation results. Next, we will continue analysing more subjects to compare the results between subjects. Finally, we would like to provide an early detection for the subjects to know if they have depression using EEG machine.

\section{REFERENCES}

[1] K. Kramlinger, Mayo Clinic (2001). Mayo Clinic on depression. Rochester, Minn.: Mayo Clinic.

[2] G. Andersson (2006). Internet-based cognitive-behavioural self-help for depression. Expert Rev Neurother; 6: 1637-1642

[3] S. Ali AlMejrad. (2010). Human Emotions Detection using Brain Wave Signals: A Challenging, European Journal of Scientific Research, 44 (4), 640-659

[4] A.R. Desai, (2017). EEG-based evaluation of cognitive and emotional arousal when coding in different programming languages. University of Oulu

[5] C. Gabriel and Luiz Moreno, Robson, (2018) Netlab MLP Performance Evaluation for Pattern Recognition in Myoletric Signal. Procedia Computer Science.

[6] Oei, Tian Po and Sawang, Sukanlaya and Goh, Yong Wah and Mukhtar, Firdaus, (2013) Using the Depression Anxiety Stress Scale 21 (DASS-21) across cultures. International journal of psychology: Journal international de psychologies: 48.

[7] R. Khosrowabadi, R.; Quek, C.; Ang, K.K.; Wahab, A., (2014). "ERNN: A Biologically Inspired Feedforward Neural Network to Discriminate Emotion from EEG Signal", IEEE Transactions on Neural Networks and Learning Systems, (25), 609 - 620, March 2014.

[8] Wahab, A., Kamaruddin, N., M. Nor, N., Abut, H., "Pre- and Postaccident Emotion Analysis on Driving Behavior", Smart Mobile In-Vehicle Systems, 225-239, 2014 